\title{
Geomagnetic jerks characterization via spectral analysis
}

\author{
B. Duka ${ }^{1}$, A. De Santis ${ }^{2,3}$, M. Mandea ${ }^{4}$, A. Isac ${ }^{5}$, and E. Qamili ${ }^{2}$ \\ ${ }^{1}$ Faculty of Natural Sciences, University of Tirana, Tirana, Albania \\ ${ }^{2}$ Istituto Nazionale di Geofisica e Vulcanologia, Roma, Italy \\ ${ }^{3}$ Universita' G. D'Annunzio, Chieti, Italy \\ ${ }^{4}$ Centre National d'Etudes Spatiales, Paris, France \\ ${ }^{5}$ Geological Institute of Romania, Bucharest, Romania \\ Correspondence to: B. Duka (bejo.duka@unitir.edu.al)
}

Received: 12 December 2011 - Published in Solid Earth Discuss.: 20 January 2012

Revised: 4 April 2012 - Accepted: 12 April 2012 - Published: 9 May 2012

\begin{abstract}
In this study we have applied spectral techniques to analyze geomagnetic field time-series provided by observatories, and compared the results with those obtained from analogous analyses of synthetic data estimated from models. Then, an algorithm is here proposed to detect the geomagnetic jerks in time-series, mainly occurring in the eastern component of the geomagnetic field. Applying such analysis to time-series generated from global models has allowed us to depict the most important space-time features of the geomagnetic jerks all over the globe, since the beginning of XXth century. Finally, the spherical harmonic power spectrum of the third derivative of the main geomagnetic field has been computed from 1960 to 2002.5, bringing new insights to understand the spatial evolution of these rapid changes of the geomagnetic field.
\end{abstract}

\section{Introduction}

Studies of discrete time-series of physical quantities are widely interesting not only for their forecasting, but also for defining both nature and behavior of the underlying physical phenomena. Different methods of time-series analyses have been used to study the geomagnetic field which is, at all times, subject to temporal variations on a wide range of time scales. Most of the rapid variations are linked to the solar activity and solar variability (many different forms include solar flares, coronal mass ejections, solar wind sector boundaries, coronal hole streams), as well as the Earth's environment (e.g. interactions between the solar wind and the core field). Most of the slow variations are generated in the outer fluid core (changes in the fluid flow). The temporal variations in the geomagnetic field cover a huge range of time-scales, from seconds to hours (external in origin), from months to decades (overlapping between external and internal changes), or from millennial to reversals (internal variations). Here, we focus on the analysis of time variations of geomagnetic field of the intermediate-range (longer than a day, shorter than a decade), especially on time variations in range of months, known as geomagnetic jerks.

The geomagnetic jerks (Courtillot et al., 1978), can be defined as sudden changes (a V-shape like change) in the slope of the secular variation (SV), i.e. the first time derivative of the Earth's magnetic field, or an abrupt (step-like) change in the secular acceleration (SA), i.e. the second time derivative. As a very first approximation, the secular variation can be described as a set of linear changes over some years to some decades, separated by geomagnetic jerks occurring on a time-scale of a few months when the nearly constant secular acceleration changes sign (and, eventually, its magnitude) abruptly. For a more detailed characterization of geomagnetic jerks, we have to consider the findings of Alexandrescu et al. (1996). Indeed, when the wavelet technique has been applied to series of monthly means, it appears that the event reveals a singular behavior with a fractional derivative close to an order 1.5. This interesting behavior would be useful to analyze the geomagnetic jerks at the place of their origin, indeed the top of the core. However, in the present analysis, we consider geomagnetic jerks more conventionally as singularities characterized by discontinuities of an integer derivative (i.e. a second order). 
Nowadays, it is almost accepted that geomagnetic jerks are internal in origin, i.e. they are produced by fluid flows at the top of the outer core. Some attempts to explain their physical origin have been done. One of them, found in Bloxham et al. (2002), explains jerks origins by a combination of a steady flow and a simple time-varying, axisymmetric, equatorially symmetric, toroidal zonal flow, consistent with sustained torsional oscillations in the Earth's core.

Usually, geomagnetic jerks are particularly visible in the eastward component $(\mathrm{Y})$, which is supposed to be the least affected by the external fields (Mandea et al., 2010). More likely affected by external field contaminations are the northward component $(\mathrm{X})$ and, slightly less, the vertical downward component $(\mathrm{Z})$. An easy method to determine the epoch when a geomagnetic jerk occurs is to approximate secular variation time-series by straight lines and to consider the intersection point of such lines as the date of an event (Chau et al., 1981; Stewart and Whaler, 1992). During the last two decades, more powerful methods to detect geomagnetic jerks and to estimate their location and duration have been developed. For example, the continuous wavelet analysis has been largely applied to the monthly mean series provided by different geomagnetic observatories (Alexandrescu et al., 1995; Alexandrescu et al., 1996; Chambodut et al., 2005), or a statistical time-series model has been used to analyze monthly means of the geomagnetic eastward component at different observatories (Nagao et al., 2003). More recently, Pinhero et al. (2011) have modeled the secular variation by two straightline segments around the time occurrence of known jerks (1969, 1978, 1991, 1999). Using the least-squares method and L-norm method, error bars in the jerk occurrence times have been estimated for almost all ground observatories. According to their results, the 1969,1978 and 1991 jerks are globally detected with an occurrence time covering a large interval for different observatories and different components, with mean errors of 1.7 years (X component), 1.1 years $(\mathrm{Y}$ component) and 1.5 years ( $\mathrm{Z}$ component).

In this work, we have used three different methods to study time-series of geomagnetic field components and secular variations, with particular attention to the $\mathrm{Y}$ component. All methods are essentially spectral analyses. Two of them, the Short Time Fourier Transform (STFT) and Discrete Wavelet Transform (DWT), derive directly as natural developments of Fourier Analyses, while the third one is a spatial spectral analysis in spherical harmonics performed at different successive epochs. The first two methods are essentially single-station time-series analyses, while the third one is a global spherical harmonic analysis. In this paper, we present the results of applying these methods on time-series of geomagnetic fields of different observatories or time-series of synthetic data generated from different models. Thereafter, we discuss the results and conclude.

\section{Data: observed and model-based temporal series}

Before presenting the applied methods, we describe the used data. The first dataset, based on real data, is composed of time-series of geomagnetic field components recorded by several geomagnetic observatories. They are chosen to be longer than 50 years and located as far as possible from each other. In addition, some synthetic data have been generated by means of specific function (see Sect. 3.1.2) or by specific composition (see Sect. 3.2.2) that simulate geomagnetic jerks, in order to optimize the real data processing. We have then generated time-series of geomagnetic field components, secular variation or secular acceleration from two geomagnetic field models described below, for a regular (uniform) grid of points over the Earth, allowing investigation of specific, large scale behavior of jerks over the globe. We have also used one of these models to investigate the third derivative of the Gauss coefficients.

\subsection{Observatory data}

In this work, we have considered several observatories: Alibag (ABG), Apia (API), Chambon La Foret (CLF), Eskdalemuir (ESK), Gnangara (GNA), Hermanus (HER), Huancayo (HUA), Kakioka (KAK), Lerwick (LER), Pilar (PIL), Sitka (SIT), Vassouras (VSS), for which hourly means have been downloaded ${ }^{1}$. From the original hourly means of these observatories, their monthly mean values series have been calculated.

In addition, a long and typical time-series of the geomagnetic field has been recorded at Niemegk Observatory (before 1932 observations were made nearby at Potsdam, then Seddin). The annual means series of $\mathrm{X}, \mathrm{Y}, \mathrm{Z}$ components and the differences of sequential values $(\Delta \mathrm{X} / \Delta t, \Delta \mathrm{Y} / \Delta t, \Delta \mathrm{Z} / \Delta t$, with $\Delta t=1$ year) are presented in Fig. 1. The monthly means of $\mathrm{X}, \mathrm{Y}, \mathrm{Z}$ components show the same behavior as the annual means, but the differences of sequential values $(\Delta \mathrm{X} / \Delta t$, $\Delta \mathrm{Y} / \Delta t, \Delta \mathrm{Z} / \Delta t$, with $\Delta t=1 \mathrm{month})$, show that they are bearing a great amount of noise not filtered from the signal (Fig. 1). A glance at these plots stimulates two remarks. First, the same field component has the same behavior in both timeseries, however, mainly for the X component, the noise level is higher in the monthly means. Second, the secular variation (first differences of the component values) shows changes in its trend, better observed in the annual curves then in the monthly ones.

Amongst the considered observatories, 4 of them, indicated in Table 1, have been chosen as representative for our analyses. These observatories have been selected because their continuous recordings over more than 50 years and their location at different latitudes and longitudes.

\footnotetext{
${ }^{1}$ http://spidr.ngdc.noaa.gov/spidr/
} 

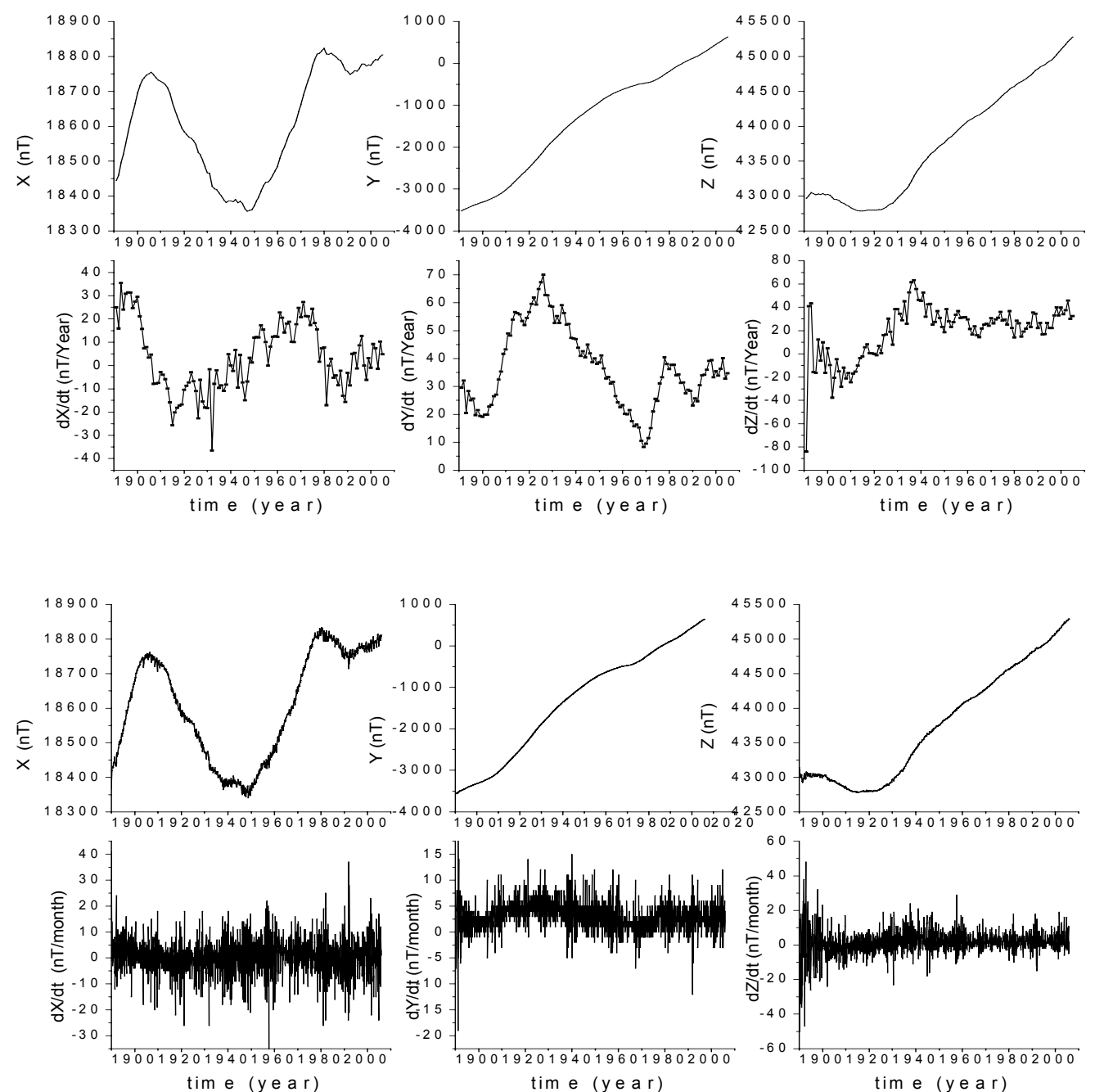

Fig. 1. Annual (upper) and monthly (lower) mean series of X, Y, Z components and their numerical derivatives (differences of the sequential values).

Table 1. The geomagnetic observatories that have been chosen as representatives for analyses.

\begin{tabular}{llll}
\hline IAGA code & Latitude & Longitude & Altitude $(\mathrm{m})$ \\
\hline API & $-13^{\circ} 48^{\prime}$ & $188^{\circ} 13.2^{\prime}$ & 4 \\
HER & $-34^{\circ} 25.2^{\prime}$ & $19^{\circ} 13.8^{\prime}$ & 26 \\
KAK & $36^{\circ} 13.8^{\prime}$ & $140^{\circ} 11.4^{\prime}$ & 36 \\
NGK & $52^{\circ} 4.2^{\prime}$ & $12^{\circ} 40.8^{\prime}$ & 78 \\
\hline
\end{tabular}

\subsection{Geomagnetic models}

Time-series of the geomagnetic field components, their secular variation and acceleration are generated from two models, CM4 (Sabaka et al., 2004) and Gufm1 (Jackson et al., 2000).

The CM4 model (Sabaka et al., 2004) entails the parameterisation and co estimation of fields associated with the major magnetic field sources in the near-Earth regime from field measurements taken from ground-based observatories and satellite missions (POGO, Magsat, Ørsted, CHAMP). It supplies the local X, Y, Z components of the $\mathbf{B}$ magnetic field vector from the main, lithosphere, primary and induced magnetosphere, primary and induced ionosphere, and toroidal field sources. Two evaluations of the main field are accommodated per two given spherical harmonic degree ranges for the span period 1960-2000 (http://core2.gsfc.nasa.gov/CM/ CM4_A.html). The capacity of this model to represent geomagnetic jerks has already been investigated (Sabaka et al., 
2002; Chambodut and Mandea, 2005). Here, we use the timeseries of the third derivative of Gauss coefficients (19602002.5) to study any possible relation between maxima of the corresponding spherical harmonic power spectra and geomagnetic jerk occurrence.

The Gufm1 model (Jackson et al., 2000), is based on a massive compilation of historical observations of the geomagnetic field (from 1590 to 1990). For the period before 1800 , more than 83000 individual observations of magnetic declination were recorded at more than 64000 locations; more than 8000 new observations came from the 17th century alone. Since no intensity data are available prior to 1840 , the axial dipole component is linearly extrapolated back before this date. The time-dependent field model constructed from this dataset is parameterised spatially in terms of spherical harmonics and temporally in B-splines, using a total of 36512 parameters (http://jupiter.ethz.ch/ cfinlay/gufm1. $\mathrm{html}$ ). This model has been used to generate monthly series of $\mathrm{X}, \mathrm{Y}, \mathrm{Z}$ components and their secular variation on a regular grid on the Earth's surface.

\section{Methods: characteristics and application to datasets}

\subsection{Short Time Fourier Transform (STFT)}

\subsubsection{STFT - definition and representation}

It is well known, that the Fourier analysis breaks down a signal into constituent harmonics of different frequencies. For regularly sampled data, Fourier analysis is performed using the discrete Fourier transform (DFT).

Using the Fourier transform of a signal, it is impossible to indicate when particular events (such as drifts, trends, abrupt changes, etc.) appear within the time-series. This deficiency can be corrected by applying the Fourier transform only to small sections of the signal at successive times, a technique called windowing the signal (Gabor, 1946) or the Short Time Fourier Transform (STFT) (see Appendix A). The STFT maps a signal into a two-dimensional function of time and frequency and can provide information about both time and frequency, thus characterizing any eventual irregularity (i.e. a spectral feature which is different from the typical behavior of the signal under scrutiny) present in the analyzed timeseries.

In order to detect particular events in long time-series of the geomagnetic field components, secular variation or secular acceleration, we have used the "specgram" function of Matlab7 software which computes the windowed discretetime Fourier transform of a signal using a sliding window (Matlab release notes, 2004 - see Appendix A). The spectrogram is the magnitude of this function expressed in decibels (dB). Different kinds of windows have been tested, with different lengths and different overlaps, providing a sampling frequency: $f_{s}=1$ (month $^{-1}$ or year ${ }^{-1}$ according to the kind of analysis). To avoid a flat spectrum in the case of geomagnetic field components, an average value of series is subtracted from each input data.

The most used windows have a Gaussian-like form (e.g. Blackman, Bohman, Chebyshev, Gaussian, Hamming, Hann, Parzen windows), and we notice that the results of spectrogram analyses almost do not depend on the form of the window, but depend on the windows length (window parameter). The shorter this length is, the smoother the spectrum. By testing different lengths, we have tried to find the most appropriate one to identify the known geomagnetic jerk occurrence.

\subsubsection{SFTF - applied to a synthetic signal}

Mathematically, the jerk events are discontinuities (breakdowns) of the second derivatives of the geomagnetic field components. To test the real effectiveness of different techniques, we have considered a synthetic signal which has such breakdowns in its second derivative. Then, we have taken the advantage of the found results to apply the same processing scheme to the real data.

We consider the following synthetic signal as defined in the interval $-0.5 \leq t \leq 0.5$

$f(t)=\left\{\begin{array}{l}\exp \left(-40 \cdot t^{2}\right) \text { for }-0.5 \leq t<0 \\ \exp \left(+5 \cdot t^{2}\right) \text { for } 0 \leq t \leq 0.5\end{array}\right.$,

and sampled at every $\Delta t=10^{-3}$. We have actually rescaled the temporal abscissa as time $=500+t \cdot 1000$, i.e. in the interval of time 0-1000 (Fig. 2). We chose such a signal because it and its first derivative have a smooth behavior during the whole interval, but its second derivative breaks down exactly at time $=500(t=0)$ showing a jerk-like behavior. One cannot detect any breakdown in the signal plot (Fig. 2, upper left) and in the spectrogram of the signal (Fig. 2 down left). The spectrogram of the first differences shows a clear breakdown close to the real one at time $=500$ (Fig. 2, down right). By trials, we have chosen the Matlab specgram function parameters $\left(n f f t=1000, f_{s}=1\right.$, Hamming window length $=12$, overlaps $=10$ ) as the most effective ones to detect the breakdown of the second derivative. Using a window parameter 12 does not mean that the data length in the input to the FFT is a 12 multiple, but a longer data, with a weighting tapering towards zero at the ends. As the vast majority of these data are zero, the STFT method produces heavily smoothed plots as it can be seen in Fig. 2 .

\subsubsection{SFTF - applied to annual series}

We present here some results of the SFTF analyses, firstly applied to NGK series of 116 averaged annual means (from 1890 to 2005). In case of X, Y, Z component series, from the original data the average value of each series is subtracted correspondingly. In the spectrograms of these field components series and their first differences (see Fig. 3) the same 

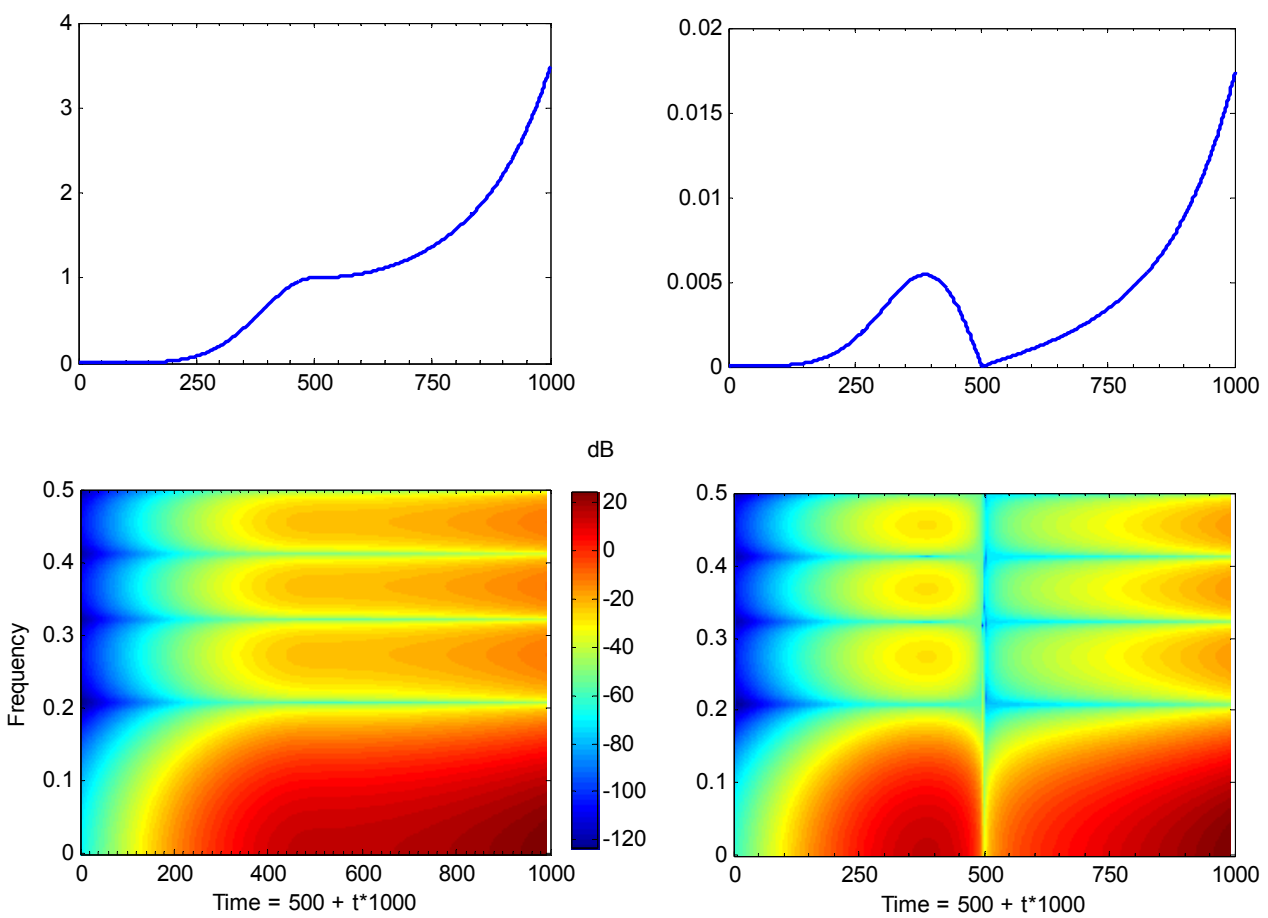

Fig. 2. The signal (1) (up left) sampled at every $\Delta t=10^{-3}$ with the temporal abscissa rescaled as time $=500+t 1000$, the spectrogram of the signal (down left), the first difference series (upper right) and its spectrogram (down right). The units are arbitrary.

kind of window (Hamming), the same lengths of window (12 values) and overlaps (10 values) are used. The spectrograms of different components show particular events at different epochs, most of them not corresponding to the known geomagnetic jerks found in literature (e.g. Mandea et al., 2010). The spectrograms of the first differences of the consecutive annual values, show the evidence of particular events likely to be the geomagnetic jerks especially in the case of the $\mathrm{Y}$ component. In this case, there is a clear evidence of a special event around 1969, identified by a large separation of different patterns of spectrum, that corresponds to the first noted geomagnetic jerk. One can also find some evidence of the geomagnetic jerks of 1901, but little evidence of other known events. For instance, there is very little evidence of an event in the middle of two known close events of 1991 and 1999, that are difficult to be identified by this spectrogram. There is a large sector of high values of power $(\mathrm{dB})$ at low frequency contents which peak (higher frequency) corresponds to the large maximum event of 1925 (compare the graph in the middle of second row of Fig. 1 with Fig. 3d).

\subsubsection{SFTF - applied to monthly series}

The first differences of monthly means of the geomagnetic field components represent very irregular and noisy signals (Fig. 1). In order to minimize this noise, i.e. to reduce annual variation mainly produced by the external field variations (ionospheric and magnetospheric variations), we have applied a moving average approach (Olsen and Mandea, 2007 ) to produce a 12 -month running mean every month, thus we have obtained monthly secular variation values by subtracting the mean of 12 earlier consecutive monthly values from the mean of the next 12 monthly values of the geomagnetic field component (Mandea et al., 2000).

Applying the "specgram" function to the monthly series of 12-months running mean secular variation of $\mathrm{Y}$ component of NGK observatory with the same kind of window, the same length and overlaps as in the case of annual series, we have obtained the spectrogram shown in Fig. 4. We can detect the geomagnetic jerks around the years 1901, 1969 and hardly that of 1990, which are however better underlined here than in the case of annual average differences. The reason is that the technique provides better results when applied to longer datasets.

In order to improve further the results of the spectrogram method, we have considered an efficient technique of secular variation de-noising that uses the wavelet decomposition of signals. Some different technique of de-noising secular variation data from the external contamination, has been recently applied by Wardinski and Holme (2011), better physically justified than the one used here. We prefer to consider hereafter the wavelet decomposition technique being more efficient to our aims. 

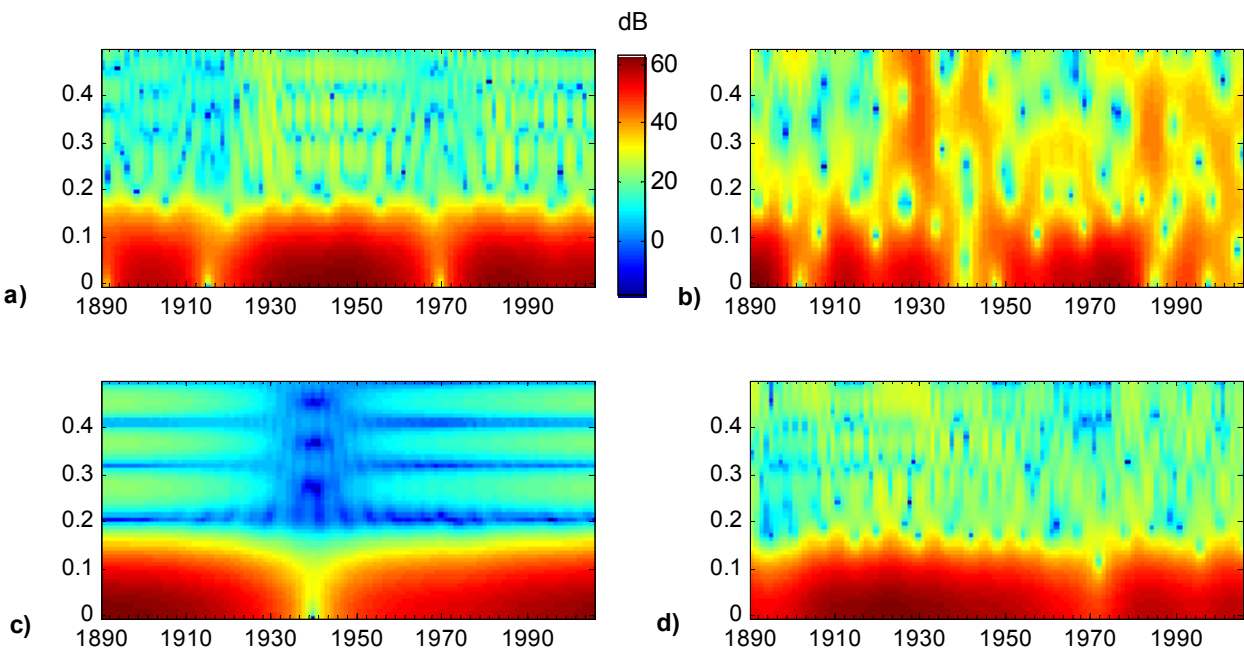

d)
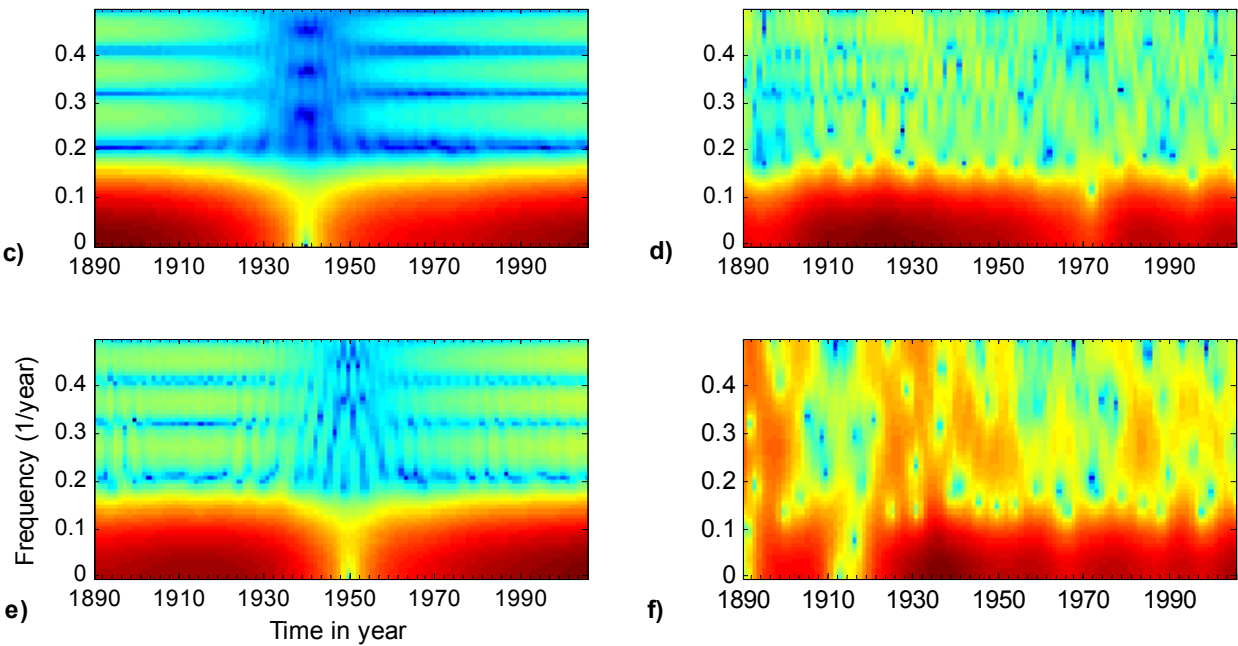

Fig. 3. Spectrograms of annual means of the geomagnetic field components and their secular variation (first differences) for NGK observatory (1890-2005): (a) X component, (b) X secular variation, (c) Y component, (d) Y secular variation, (e) Z component, (f) Z secular variation.

\subsection{Discrete Wavelet Transform (DWT)}

\subsubsection{DWT - definition and representation}

Wavelet analysis represents a windowing technique with variable-sized regions, normally with long time intervals providing more precise low-frequency information, and shorter time intervals with high-frequency information. Wavelet analysis is capable of revealing aspects of data like trends, breakdown points, discontinuities in higher derivatives, and self-similarity. It is also used to compress or de-noise a signal without appreciable degradation (e.g. Kumar and Georgiu, 1994). For the self-consistency of this paper, a few specificities of the wavelet analysis are summarized in the following, whereas full information can be found, for example, in Grossmann et al. (1987), Meyer (1992, 1993) and Holschneider (1995), Misiti et al., (2007), Brockwell et al (2009) to cite a few.

The wavelet analysis is the breaking up of a signal $s(t)$ into scaled and shifted versions of the original (or mother) wavelet function $\Psi(t)$. If a function $\Psi$ is continuous, has null moments, decreases quickly towards 0 when $t$ tends towards infinity, or is null outside a segment of $R$, it is a likely candidate to become a wavelet. The wavelet decomposition consists of calculating a "resemblance coefficient" between the signal and the wavelet located at position $b$ and of scale $a$. The family of such coefficients $C(a, b)$ depends on two indices $a$ and $b$ (Kumar and Georgiu, 1994):

$C(a, b)=\int_{R} s(t) \frac{1}{\sqrt{a}} \Psi\left(\frac{t-b}{a}\right) d t$.

Scaling (dilating) a wavelet simply means stretching (or compressing) it by a scale factor $a$. Shifting (translating) a wavelet simply means delaying (or hastening) its onset. In the Continuous Wavelet Transform (CWT), the set to which $a$ and $b$ belong is: $a \in R^{+}-\{0\}, b \in R$. In the Discrete Wavelets Transform (DWT), the scale parameter $a$ and the location parameter $b$ are discrete, usually based on powers of two: $a=2^{j}, b=k \cdot 2^{j},(j, k) \in Z^{2}$ (so-called dyadic scales and positions).

Defining:

$\psi_{j, k}(t)=\frac{1}{\sqrt{2^{j}}} \psi\left(\frac{t-k 2^{j}}{2^{j}}\right)=2^{-j / 2} \psi\left(2^{-j} t-k\right)$,

it is possible to construct a certain class of wavelets $\Psi(t)$ such that $\Psi_{j, k}(t)$ are orthonormal, i.e. the wavelets are orthogonal to their dilates and translates:

$\int \psi_{j, k}(t) \psi_{j^{\prime}, k^{\prime}}(t) d t=\delta_{j j^{\prime}} \delta_{k k^{\prime}}$. 

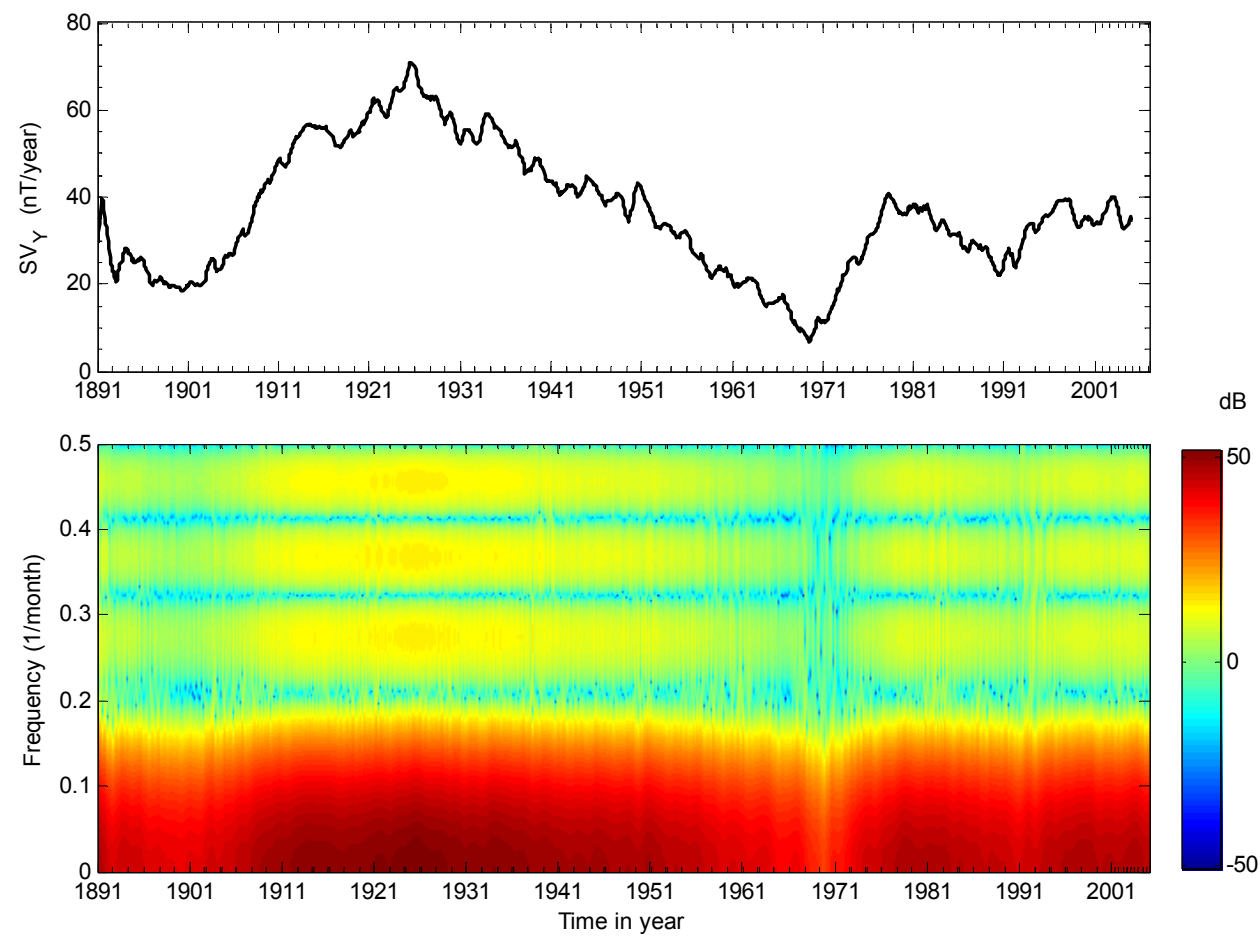

Fig. 4. The monthly series of $\mathrm{SV}_{Y}$ (upper panel) produced by 12-month running mean for NGK observatory (1890-2005) and its spectrogram (bottom panel).

All such functions $\Psi_{j, k}(t)$ form a complete orthonormal basis for all functions $s(t)$ that have finite norm, i.e. the time signal $s(t)$ is expressed by the coefficients of discrete wavelet decomposition $C(j, k)$ as:

$$
\begin{aligned}
& s(t)=\sum_{j \in Z} \sum_{k \in Z} C(j, k) \psi_{j, k}(t), \text { where } C(j, k)=\left\langle s, \psi_{j, k}\right\rangle \\
& \equiv \int s(t) \psi_{j, k}(t) d t
\end{aligned}
$$

Let us fix $j$ and sum on $k$. A detail $d_{j}$ is then the function:

$d_{j}(t)=\sum_{k \in Z} C(j, k) \psi_{j, k}(t)$.

The signal is the sum of all the details:

$s=\sum_{j \in Z} d_{j}$.

Let us take now a reference level called $J$. There are two sorts of details. Those associated with indices $j \leq J$ correspond to the scales $a=2^{j} \leq 2^{J}$ which are the fine details. The others, which correspond to $j>J$, are the coarser details. We group these latter details into:

$a_{J}=\sum_{j>J} d_{j}$.

which defines what is called an approximation of the signal $s$. We have just created the details and an approximation. The equality:

$s=a_{J}+\sum_{j \leq J} d_{j}$,

means that the signal $s$ is the sum of its approximation $a_{J}$ and of its fine details $d_{j}$.

Wavelet Toolbox of Matlab software (http://www. mathworks.com/help/toolbox/wavelet/) provides a variety of the signal analysis (signal decompositions, compression, denoising, etc.) by different kinds of wavelet shapes and different levels. Aiming to detect a "rupture" in the $j$-th derivative, the selection should be a sufficiently regular wavelet with at least $j$ vanishing moments.

We have found that the kind of wavelets detecting successfully the second order derivative change in the known signal (1) is the Daubechies (Db) wavelet (Daubechies, 1992) of order 4: Db4 (see sketches of this wavelet in the Fig. 12) at level 2 of the signal decomposition:

$s=a_{2}+d_{2}+d_{1}$,

where the decomposition (see Eq. 9) ends at $J=2$. The results show anomalous values of coefficients $d_{1}$ and $d_{2}$ exactly where (time $=500)$ the signal $(1)$ has the second derivative breakdown. This breakdown is better localized by the anomalous values of $d_{1}$ coefficients. 


\subsubsection{Geomagnetic data de-noising by using DWT}

The presence of noise makes more complicated the identification of discontinuities. If the first levels of the decomposition can be used to eliminate a large part of noise, the "rupture" is sometimes visible only at deeper levels in the decomposition.

\section{Synthetic signal}

In order to define empirically the best way for applying DWT technique to the signal de-noising, we have generated a series of first differences of the several exponential spikes like (1) with different slope changes (jerk-like), respectively at time $=100,200,250,300,500,700,850$ (Fig. 5a). In order to get a signal more likely as the secular variation provided by a geomagnetic observatory, we have added to such series of synthetic signal a modified colored AR(3) noise (see Appendix B). The modifications provide a more realistic noise (more like a secular variation signal) with the amplitude of the noise of about $15 \%$ of the signal itself. The composed signal is presented in Fig. 5 b.

After applying DWT with different wavelets for signal decompositions, the most appropriate ones to get the best denoised signal are the Daubechies wavelets of order 4 and level 4 of decomposition. Such de-noised signal is presented in Fig. 5. In the bottom panel of Fig. 5 (d-f), the spectrograms of the respective signals Fig. $5(\mathrm{a}-\mathrm{c})$, are presented. The spectrograms are plotted using the same parameters of the specgram Matlab function. According to these spectrograms, we can note:

- Using an appropriate de-noising process and applying it to the composed signal (original one and noise), the obtained spectrogram is similar to that of the original signal, but allowing better than non de-noised signal (compare Fig. 5e and f) to identify the abrupt changes of slopes (breakdowns of the second derivative).

- Apart from some moderate breakdowns of the second derivative (for example at time 500 of the original synthetic signal), all breakdowns of the second derivative jerk-like can be identified by separation of the spectrogram pattern of the de-noised signal. The more abrupt the changes of the signal slope are, the more visible such separation appears.

- Looking at spectrogram of the de-noising signal, it is difficult to individuate two close and small-scale slope changes, for example those of time 200 and 250. This drawback of the SFTF method should be considered during the interpretation of the spectrograms of the denoised real secular variation.

\section{Real data}

We have applied this technique of de-noising the signal before getting the spectrogram of the secular variation for the $Y$ component $\left(\mathrm{SV}_{Y}\right)$ monthly series of the 4 observatories previously described. The obtained results are shown in Fig. 6, where the breakdowns of the spectrogram patterns correspond to the time occurrences of the jerk-like events.

Figure 6 shows that generally the spectrograms of the denoised secular variation of different observatories reflect a different behavior of the secular variation in these observatories. In the low latitude observatories (see API when compared with the others) more changes in the slope of the secular variation can be better detected than at higher latitude observatories. These changes are smaller in amplitude and longer in time and reflect long-term events, such as 1950 1954 and 1996-1998 at API observatory. From the spectrogram corresponding to API observatory, it is possible to confirm some geomagnetic jerks around 1954 and 1978. At higher latitude observatories (see NGK when compared with the others) the geomagnetic jerks noted in the original signals (as around 1925 and 1978) are difficult to be detected in the respective spectrograms. However, in NGK spectrogram, geomagnetic jerks around 1901, 1969, 1990 can be detected. Spectrograms for the middle latitude observatories (HER and KAK) indicate some different times for geomagnetic jerks. In particular, for HER observatory it is possible to note a less marked event around 1953, a stronger one around 1986 and the strongest event around 1996. The change in the slope centered in 1972 lasts here from 1968 to 1978 and can not be considered as a geomagnetic jerk signature. For the KAK observatory, we can identify a geomagnetic jerk around 2000, and hardly identify events nearly by 1953, 1962 and a double event 1968-1970. Although there are clear changes in the secular variation of KAK observatory before 1951, they might be also due to some poor quality of data in that period of time.

\subsubsection{DWT applied to the monthly series}

To determine the second derivative breakdown of the geomagnetic field components, we have applied DWT to long time-series of geomagnetic fields recorded at different geomagnetic observatories. Better results, when jerks are easily detected, have been obtained when the DWT analyses is applied to the $\mathrm{Y}$ component secular variation, calculated by the 12-month moving average. Before applying the DWT analyses, we have applied a de-noising procedure on the secular variation signal.

\section{Synthetic signal}

The composed signal (first derivative of several exponential spikes + a colored noise) is de-noised by using $\mathrm{Db}$ wavelets (order 4 and level 4) and represented in Fig. 5. The obtained 


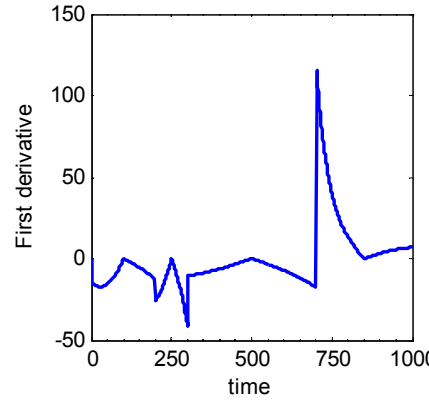

a)

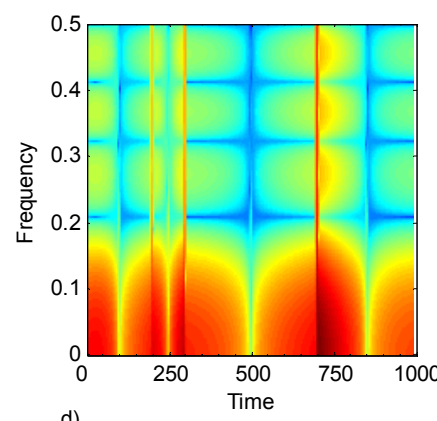

d)

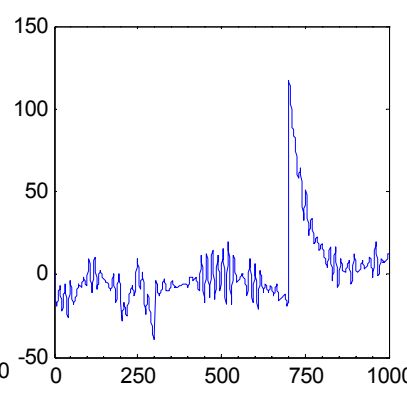

b)

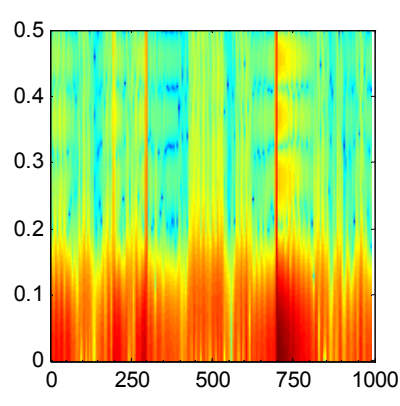

e)

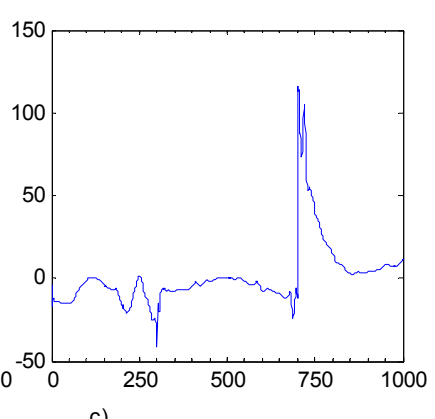

c)

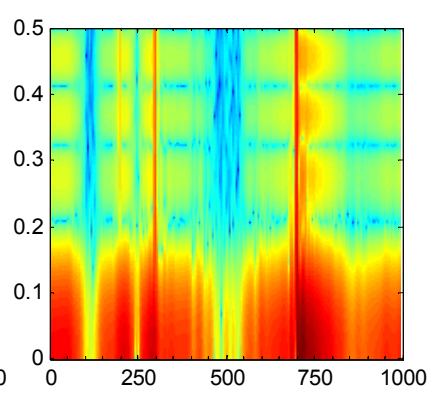

Fig. 5. (a) Synthetic signal representing a secular variation-like signal (top panel, left) composed by first differences (first derivative) of several exponential spikes; (b) Composed signal by the above synthetic signal and a colored noise; (c) The de-noised signal of the composed signal (b). The units of the signal, its derivative and time are arbitrary. Bottom panel, the spectrograms (d-f) of the respective signals (a-c).
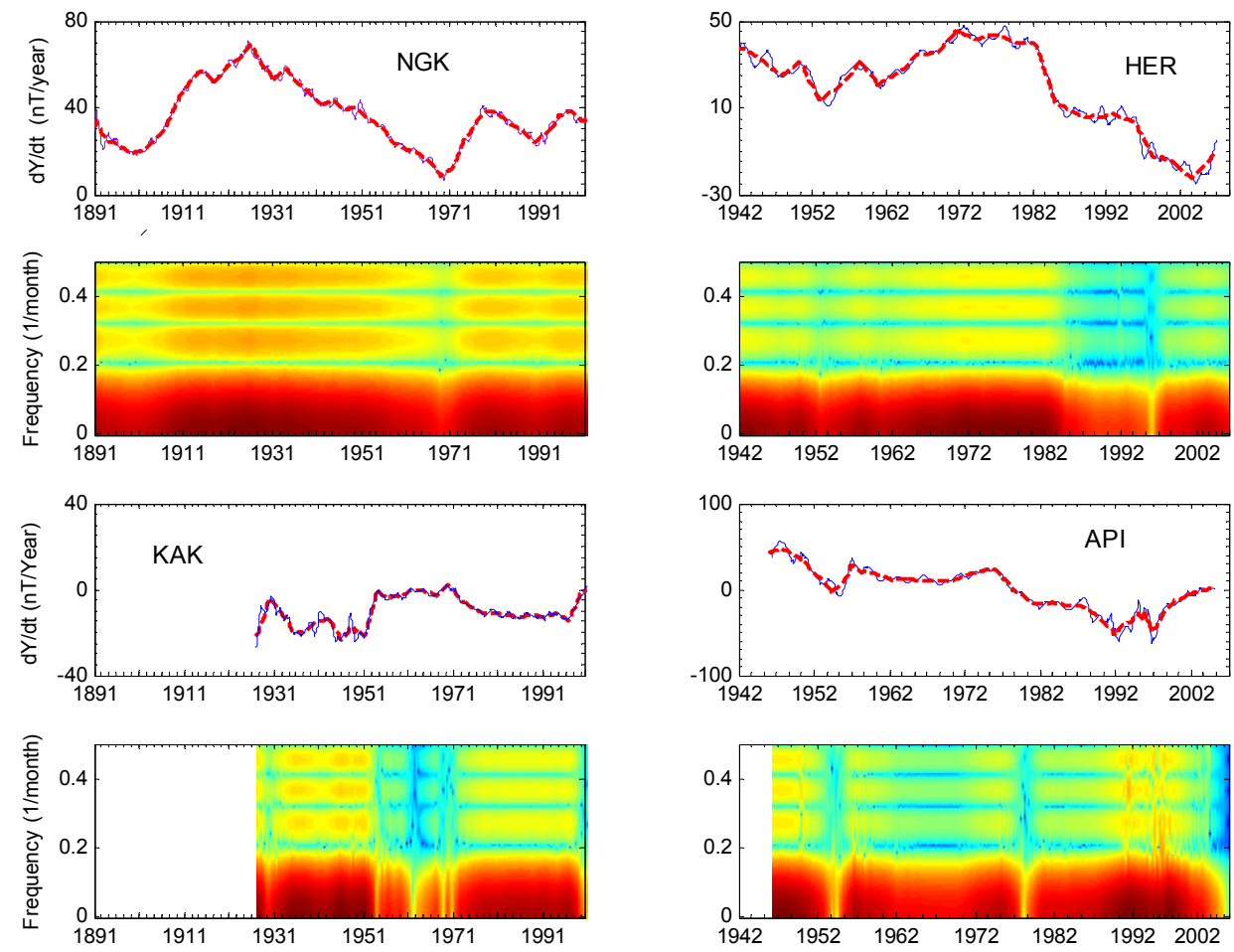

Fig. 6. The signals (blue curves) and de-noised signals (red curves) of secular variations $\left(\mathrm{SV}_{y}=\mathrm{dY} / \mathrm{dt}\right)$ of NGK, KAK, API and HER observatories and their respective spectrograms. 


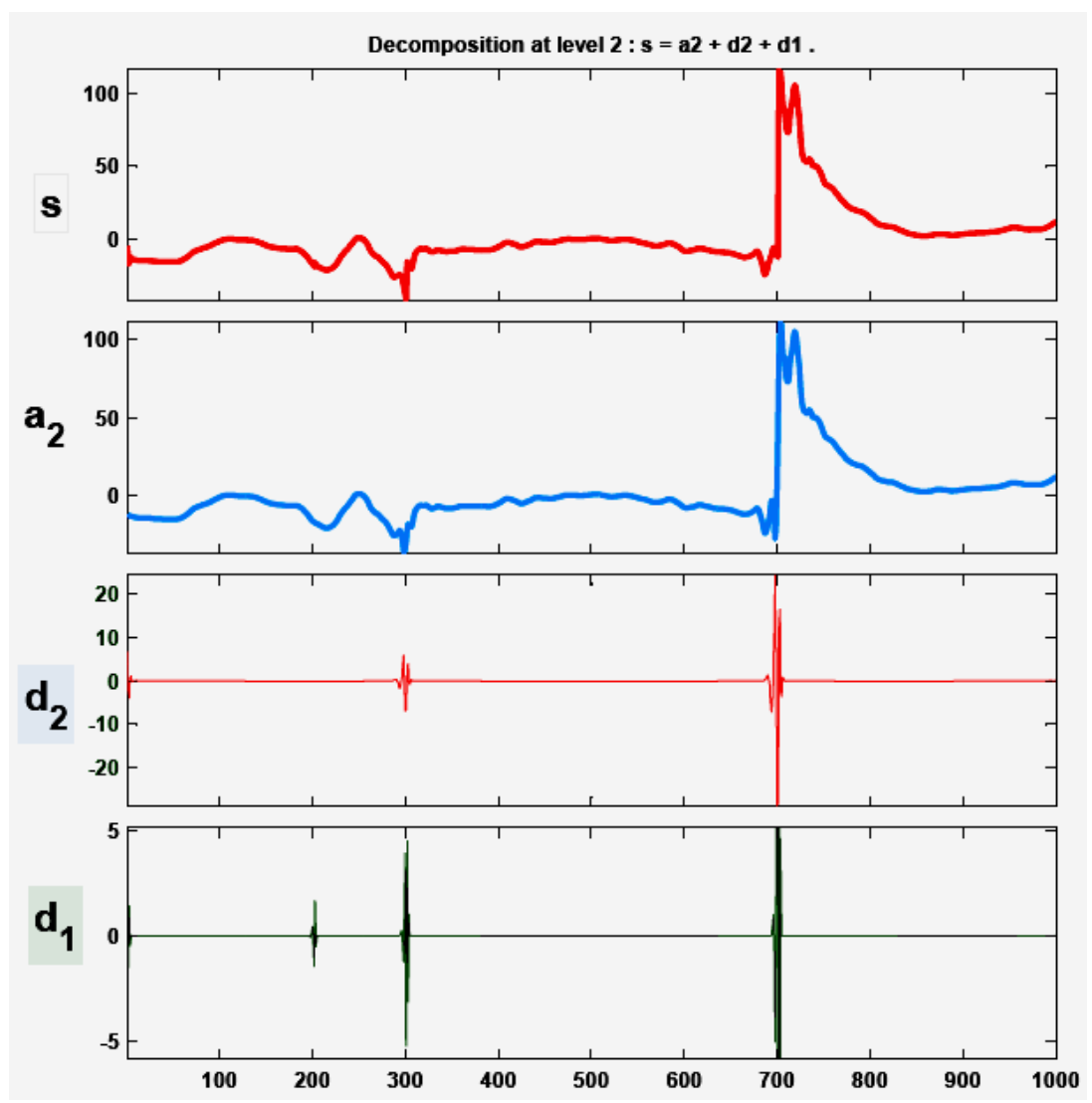

Fig. 7. The decomposition up to level 2 of the de-noised signal $s(t)$ of the synthetic composed signal (first derivative of a series of exponential spikes + colored noise). Units of the signal $s$ (approximation $a_{2}$, details $d_{1}$ and $d_{2}$ ) and the time $t$ are arbitrary.

signal is decomposed according to Eq. (9) up to level 2 (Eq. 10) by using the same Db wavelets of order 4, as is shown in Fig. 7. One can note that the maxima of the amplitude variation of $d_{1}$ and $\mathrm{d}_{2}$ coefficients correspond to the discontinuities of the first derivative of the signal, better represented by maxima of the $d_{1}$ coefficient amplitude (see Fig. 7). According to the graph scale, the $d_{1}$ coefficient values, at the breakdowns of time: $t=100,500,850$ are not visible.

\section{Real data}

Considering again the NGK observatory, a suitable denoising of the monthly series of secular variation, without distortions of the signal itself, is achieved by using the following Daubechies wavelets: Db wavelets of order 2 at decomposition level 3 or 4, Db wavelets of order 3 at decomposition level $4, \mathrm{Db}$ wavelets of order 4 at decomposition level 5 or 6 (see Fig. 8). From the previous tests we can conclude that a better way to detect particular events in such denoised series is to use the same order of Daubechies wavelets as those used for the de-noising and the level 2 of decompositions. Plotting the averaged values ( $r m s$ of the 12 values of each year) of the detail coefficients $d_{1}$ (Fig. 8) of such decompositions, the geomagnetic jerks around 1969 and 1991 are clearly detected, while in a higher decomposition level events can be noted around 1922 and 1941. This last event, not known as a regional or large-scale geomagnetic jerk, is related to changes of the secular variation slope due to several spikes close to each other.

We have then applied the same method to the monthly series of four geomagnetic observatories mentioned in Sect. 3.2.2. The results, not presented here, indicate that in order to get a reasonable de-noised signal, we have to apply different values of wavelet order and level of decompositions. The results of analyzed observatories underline different particular events, some of them corresponding to well-known geomagnetic jerks. However, we can note that from some observatory data, the presence of a large number of fringes (short spikes) in the de-noised signal makes it difficult to detect geomagnetic jerks. This particularity is linked not only to the difference in length of geomagnetic recordings and the data quality provided by different observatories, but also to the different behavior of Y secular variation over the globe. 

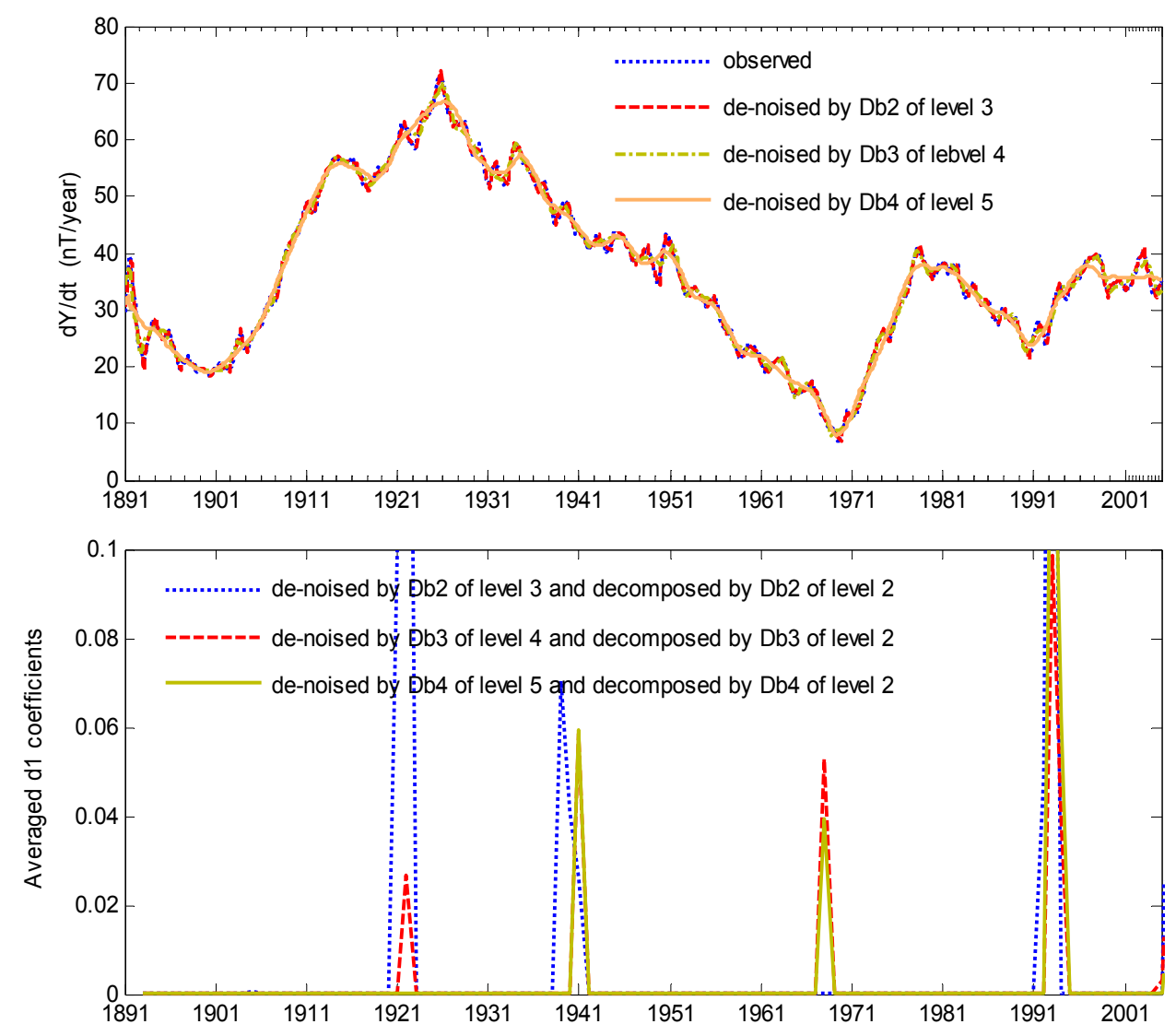

Fig. 8. De-noised $\mathrm{SV}_{Y}$ signal of NGK (up) and the respective values of averaged ( $\left.r m s\right) \mathrm{d}_{1}$ coefficients (down). The unit of $d_{1}$ coefficients are in nT/year.

\subsubsection{DWT applied to global model-based monthly series}

Accepting that the amplitude variation of the detail coefficient $\left(d_{1}\right)$ of the decomposition of the de-noised secular variation is an indicator of breakdowns in time-series of the second derivative of the geomagnetic field, we have composed the field of this amplitude (its yearly rms values) on the Earth's surface at different subsequent epochs. As the need is for long monthly series uniformly distributed over the Earth, we have used the Gufm1 model to generate them. However, an important question can arise, linked to the possible signature of the B-splines nodes of the model in the wavelet analysis of synthetic series calculated from Gufm1 model. This has been investigated (but not shown here), and the effects of jerks are much larger, with different amplitudes and occurrence times, so the B-splines nodes are not relevant for our analyses.

The Gufm1 code generates the secular variation values of the main geomagnetic field at every epoch in the range of the covered period by the model, and everywhere on the Earth. Using this code, we have firstly investigated a single location series, by generating a long monthly series (1890-1990) of
$\mathrm{SV}_{Y}$ at NGK observatory coordinates. The wavelet decomposition at level 2 by the Daubechies wavelets of order 2 is applied to this series and the rms of detailed $d_{1}$ coefficients are shown in the Fig. 9. To detect particular events, we should consider only the values of $r m s d_{1}$ coefficients larger than their mean (0.004). Here, one can identify several events, that are undeniably known as large-scale extension $(1969,1978)$, or may have a similar extension $(1913,1925)$, or seems to be more local events $(1906,1919,1949,1958)$ (Alexandrescu et al., 1996; Le Huy et al., 1998; Mandea et al., 2010). The longest event is a local one that lasts from 1942 to 1949 and has a central maximum at 1946.

Thereafter, we have applied the wavelet analyses to the monthly values of $\mathrm{SV}_{Y}$ estimated from Gufm1 on a grid of 212 points uniformly distributed over the Earth's surface, for the period 1900-1990. Each series is decomposed by Daubechies wavelets of order $2(\mathrm{Db} 2)$ at level 2, saving the coefficients of decomposition. Then, we have calculated the $r m s$ value of $d_{1}$ coefficients for every year of the considered period and plotted the field of $d_{1}$ coefficients ( $r m s$ values) for each epoch over the Earth. In Fig. 10, the fields of $d_{1}$ coefficient for a selection of epochs are presented. The deviation of the $r m s d_{1}$ coefficient from its mean value over the whole 

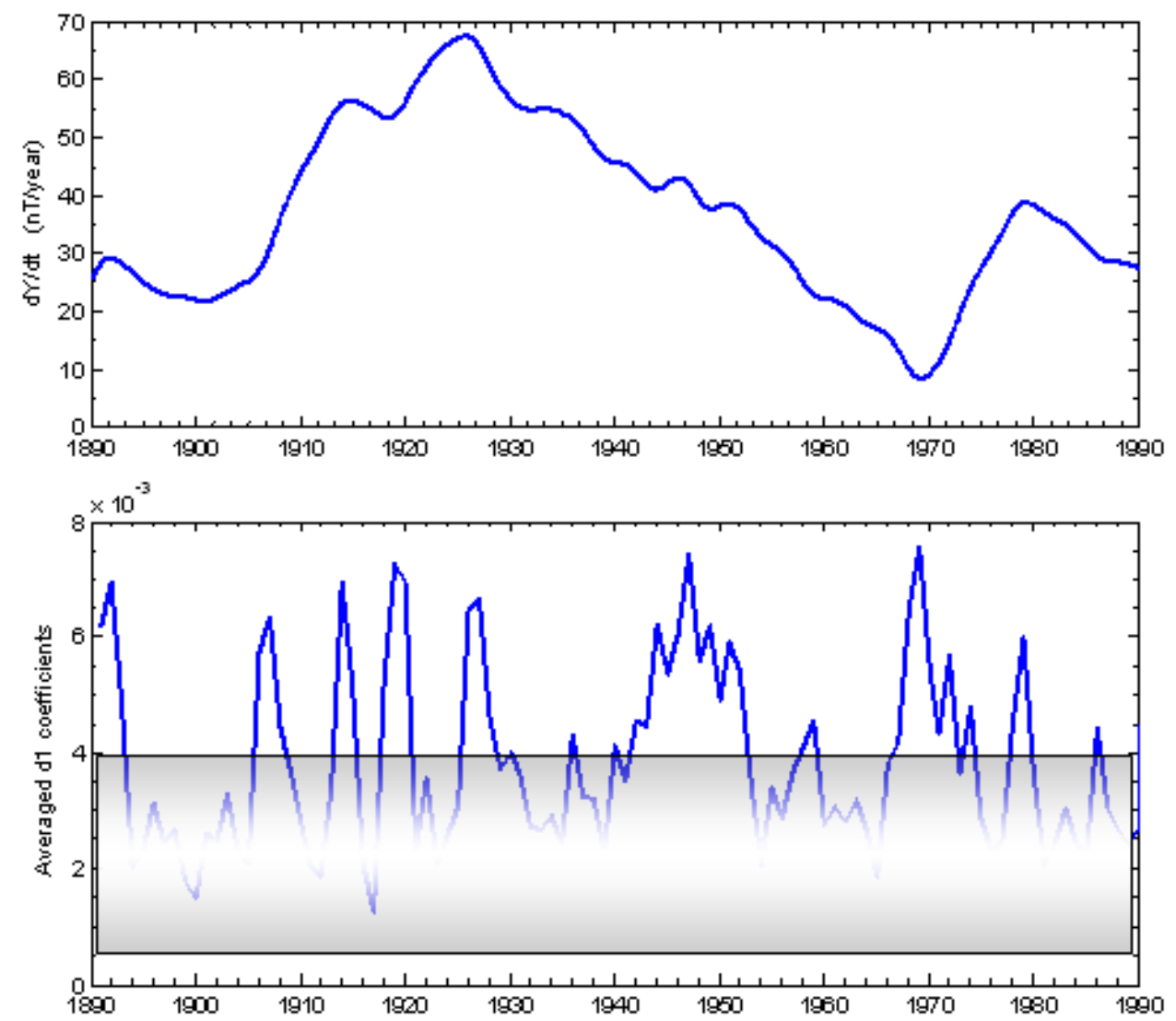

Fig. 9. Monthly series of secular variation of Y component generated by Gufm1 model at NGK Observatory coordinates for the period 1890-1990 (upper panel) and averaged $r m s d_{1}$ coefficients of the series decomposition by Db2 wavelets of level 2 . The $r m s d_{1}$ coefficients (in $\mathrm{nT} /$ year) below their mean value are covered.

period is plotted: the white areas correspond to regions where the rms $d_{1}$ values are smaller than the mean value and the black areas correspond to regions where values of the rms $d_{1}$ coefficient are greater than the maximum of the chosen scale. The plots of whole period can be seen in an additional animation (see Supplement).

Let us discuss the behavior of the $r m s d_{1}$ coefficient field, as observed from plots and animation. It is indeed possible to note a relatively strong field in 1901 , localized in four latitude belts mainly in the low and middle latitudes, which is followed by quiet fields from 1902 to 1904 . Then two small spots of a strong field appear in 1905 over the Northern hemisphere, gradually enlarged and expanded even in the Southern hemisphere in 1910, 1911, 1912 , to be reduced again in 1913.

Two other foci of strong field start in 1917, reaching a maximum the next year and being reduced to a small spot in 1920. A quiet period follows until 1925, when a strong widespread field appears, and gradually reduces over the following years, with three remaining belts getting the strongest field from 1930-1932. From 1934 to 1940 a quiet period follows, with a few small spots at different locations, however insignificant.

From 1945, a strong field wide spreads until 1949, then two large belts of longitudes characterize the period 19501954. Similar shifted belts appear again in 1960, after a period of almost quiet field from 1954-1959, reaching their maxima in 1964. Another period of quiet field reaching the smallest value almost everywhere in 1967 , is followed by a strong field reaching the maximum for the European area in 1969 and for a region situated in the Southern hemisphere in 1970.

Over the time period 1972-1978 a quiet field dominates with a few small spots of strong field near the South Pole. A strong field in 1978 is observed mainly in the large Western and Eastern longitude belts. A quiet field period ends in 1982 with the appearance of two local spots of strong field: one located around African continent and the other located in the large Western and Eastern longitudes. The latest one is faded gradually in the following years, while the first one reached maximum in 1985, moving thereafter toward the South Pole and splitting in two belts of strong field in 1987. 

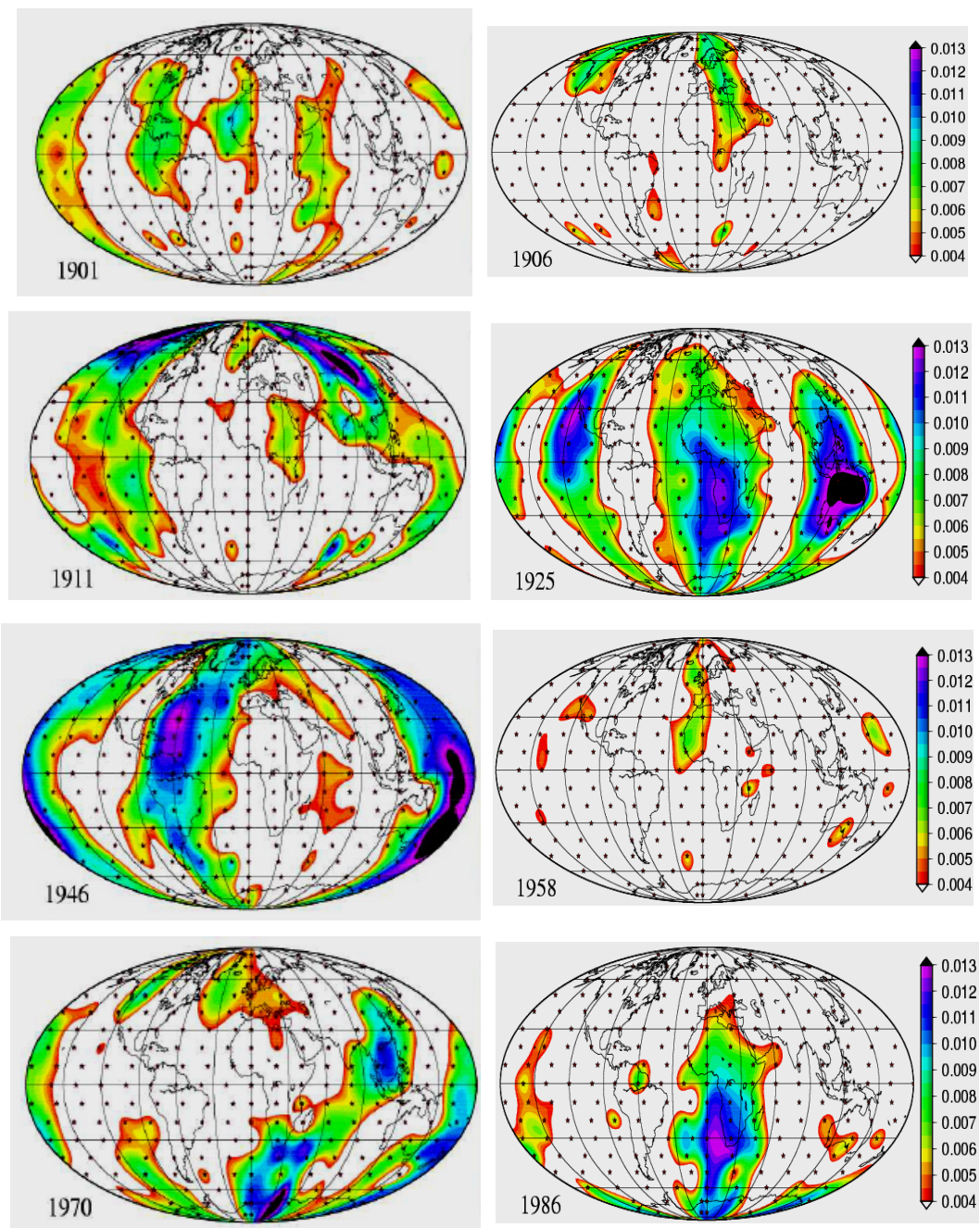

Fig. 10. An example of the field of the $r m s d_{1}$ coefficient in $\mathrm{nT} \mathrm{yr}^{-1}$, for the epochs: 1901, 1906, 1911, 1925, 1946, 1958, 1970, 1986, which are a selection from the complete animation in the Supplement.

The strongest field in 1990 must be considered with caution because of the edge effects.

\subsection{Spherical Harmonic Power Spectra (SHPS)}

The spherical harmonic analysis is a representation of the geomagnetic field potential as solution of Laplace equation. In order to detect any relation between the known jerk events and the time changes of the spherical harmonic of different degrees, we have also investigated the time variations of the Mauersberger-Lowes power spectrum terms of different degrees (Lowes, 1974, 2007) extending its definition to the third derivative of Gauss coefficients:

$R_{n}^{3 \mathrm{~d}}=\left(\frac{a}{r}\right)^{2 n+4}(n+1) \sum_{m=0}^{n}\left[\left(\dddot{g}_{n}^{m}\right)^{2}+\left(\dddot{h}_{n}^{m}\right)^{2}\right]$

with $a=6371.2 \mathrm{~km}$, the mean radius of the Earth. We have estimated the spatial power spectrum of the third derivative as a geomagnetic jerk is defined like a step-like function in the second derivative of the geomagnetic field, thus it can be somehow related to extremes in the power spectral density of the third derivative, $R_{n}^{3 \mathrm{~d}}$. We have used the CM4 model to calculate the time-series of the third derivative of the Gauss coefficients, using time increment: $\Delta t=2.5$ years, over the time-span 1960-2002.5. Fig. 11 shows plots of $R_{n}^{3 \mathrm{~d}}$ time evolution for different degrees (from $n=1$ to $n=12$ ) on the Earth's surface. The time behavior of $R_{n}^{3 \mathrm{~d}}$ at the CMB is similar, with the same relative minima and maxima, being just the same quantity scaled by a different radial ratio $(a / r)^{2 n+4}$.

According to Holme et al. (2011), the secular variation and acceleration spectra at the core-mantle boundary show strong effects of damping at degree 4 and above. This effect can be more important for the first epochs covered by a model, here, when using the CM4 model, for 1960-1962.5 timespan. This model provides the Gauss coefficients values of 

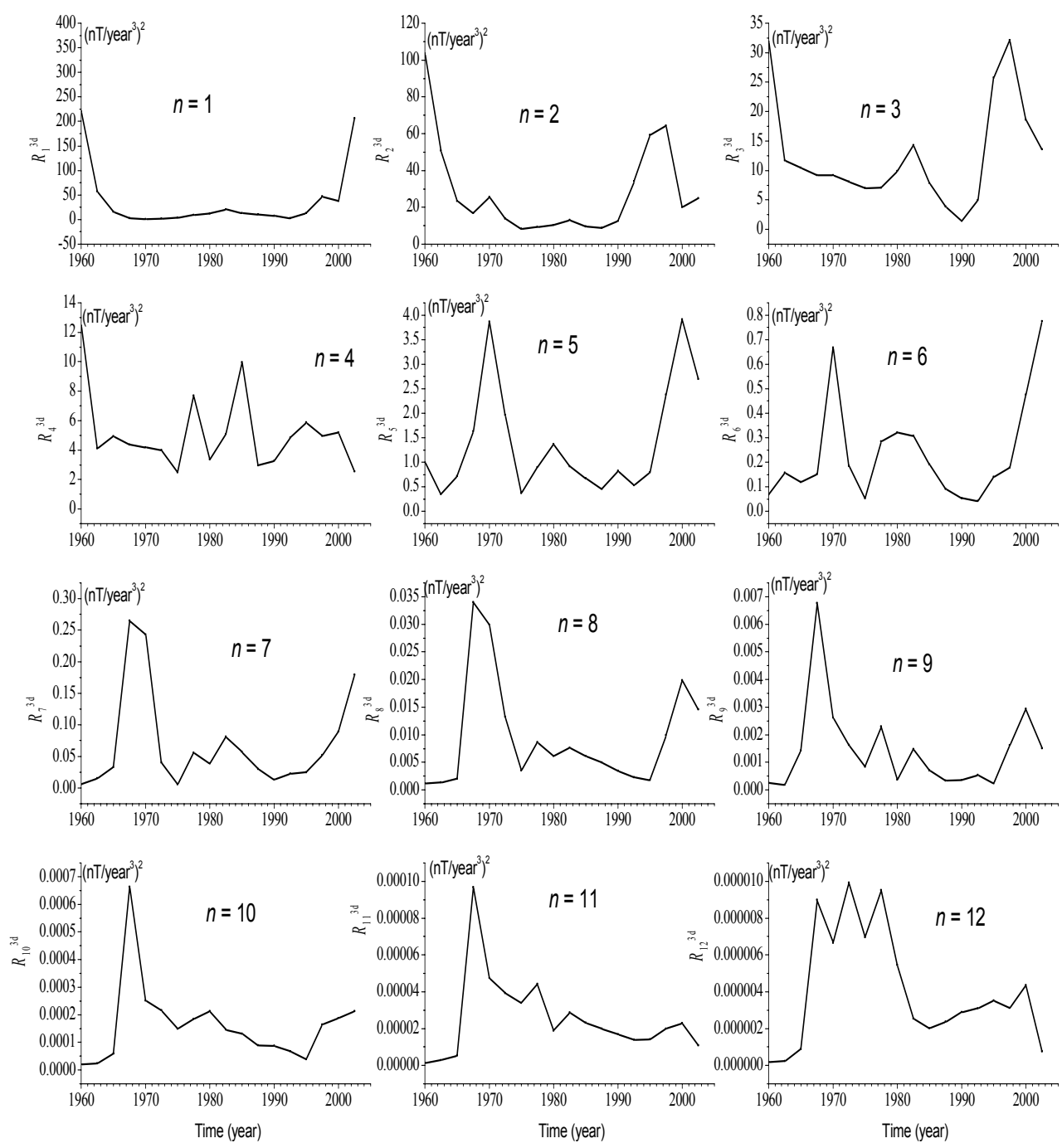

Fig. 11. Time variations of the spherical harmonic power spectra terms $\left(R_{n}^{3 d}\right)$ at the Earth's surface.
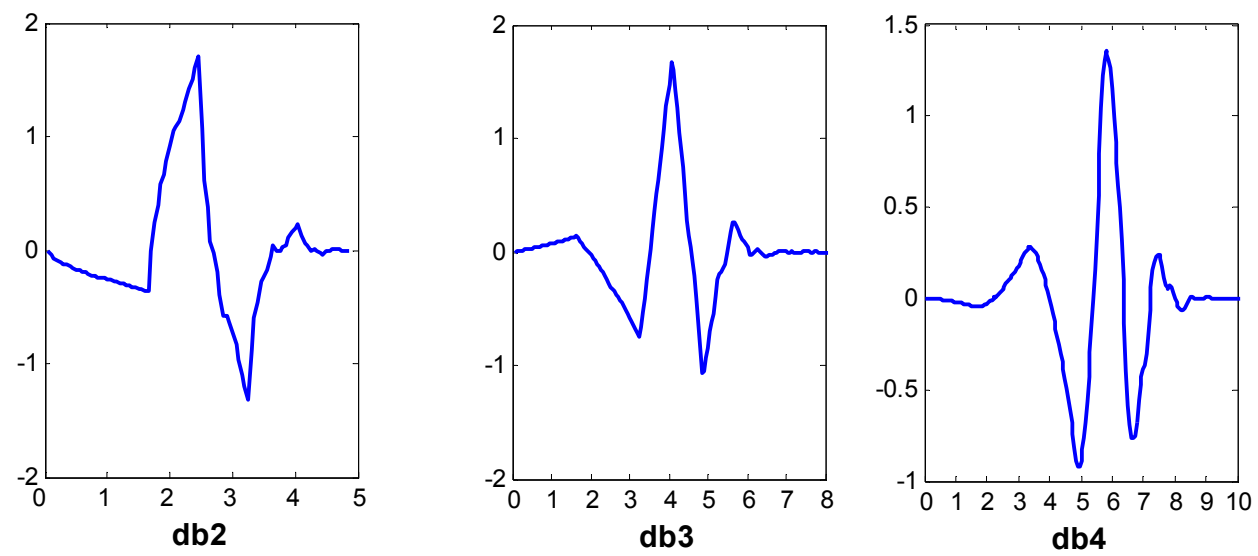

Fig. 12. Daubechies wavelet functions $\Psi(t)$ of order $2,3,4$. The units are arbitrary. 
the main field and their first, second, third and fourth derivative values of degrees from $n=1$ to $n=13$. We have checked these values and found that the Gauss coefficients values and their third derivative for the main field of CM4 model after the first couple of years of its starting time can be considered robust (at least up to $n=10$ ).

Supposing that the occurrence date of the known jerks $(1969,1978,1986,1991,1999)$ is in the middle of each epoch, the time interval (in years) of each jerk from the nearest maximum of $R_{n}^{3 t d}$ is calculated for each degree $n$. These time intervals $\Delta t_{1969}(n), \Delta t_{1978}(n), \Delta t_{1986}(n), \Delta t_{1991}(n)$, $\Delta t_{1999}(n)(n=1,2, \ldots 12)$ are presented in the second row of doubled rows of Table 2, where the sign is (-) when the jerk happened before the nearest maximum and (+) when the jerk happened after the nearest maximum. In order to weigh relatively the maxima of $R_{n}^{3 \mathrm{~d}}$ terms, we define the quantity:

$\gamma=\left(R_{n}{ }^{3 \mathrm{~d}}{ }_{\max }-R_{n}{ }^{3 \mathrm{~d}}{ }_{\min }\right) /\left(R_{n}{ }^{3 \mathrm{~d}}{ }_{\max }+R^{3 \mathrm{~d}}{ }_{\min }\right)$,

where max and min indicate that values are at the nearest maximum or minimum. The weights $\gamma$ range in the interval $[0,1]$, and they are indicated in the first row of doubled row characterizing each event. The weight of a maximum is an indicator of how clear and strong a jerk occurring near that maximum is, which may be translated in a scale information.

Averaging the time between a given jerk date and the nearest maximum for all degrees $(n=1,2, \ldots 12)$ an averaged time-interval is obtained, listed in the penultimate column of the table:

$$
\begin{gathered}
\overline{\Delta t}_{1969}=\frac{\sum_{i} \Delta t_{1969}^{i}}{12} ; \overline{\Delta t}_{1978}=\frac{\sum_{i} \Delta t_{1978}^{i}}{12} ; \overline{\Delta t}_{1986} \\
=\frac{\sum_{i} \Delta t_{1986}^{i}}{12} ; \overline{\Delta t}_{1991}=\frac{\sum_{i} \Delta t_{1991}^{i}}{12} ; \overline{\Delta t}_{1999}=\frac{\sum_{i} \Delta t_{1999}^{i}}{12}
\end{gathered}
$$

In the last column of the table the so-called "averaged scaled time-interval" is defined as the average of the time-intervals from nearest maximum when these intervals are divided by the weight of respective maximum:

$$
\overline{\Delta t}_{1969}^{s}=\frac{1}{12} \sum_{i} \frac{\Delta t_{1969}^{i}}{\gamma_{1969}} ; \overline{\Delta t}_{1978}^{s}=\frac{1}{12} \sum_{i} \frac{\Delta t_{1978}^{i}}{\gamma_{1978}} ; \ldots ;
$$

$\overline{\Delta t}_{1999}^{s}=\frac{1}{12} \sum_{i} \frac{\Delta t_{1999}^{i}}{\gamma_{1999}}$

As the weights are smaller than 1 , the divided time-intervals are increased in comparison to the respective time-intervals, and this increase is greater when the weight of maximum is smaller. In the last row of the table, the sums of time-intervals of all jerks for given degree are indicated, while the penultimate row indicate the sums of time-intervals divided by the weight of the respective maximum (scaled time-intervals).

Analyzing the values indicated on columns (degrees) of Table 2 it appears that the best coincidences of the geomagnetic jerk dates with $R_{n}^{3 \mathrm{~d}}$ maxima are found for the degrees $n=5$ and $n=9$. When we consider the sum of scaled timeintervals there is little deterioration for $n=5$, comparing with $n=9$. According to this, we can estimate a spreading behavior of the geomagnetic jerks by comparing it to the wavelengths of these harmonics $(n=5$ and $n=9)$ given by $\frac{2 \pi}{n+1 / 2} \cdot r$ (Backus et al., 1996). The obtained spatial scales of about 7300 and $4230 \mathrm{~km}$, respectively, are a confirmation of estimates from the $d_{1}$ coefficient field analysis, previously shown, where regions of the strong fields possess these spatial scales.

Analyzing Table 2 rows, in terms of geomagnetic jerks, it appears that events around 1969, 1978 and 1999 occur at the shorter averaged time-intervals from the maximums of $R_{n}^{3 \mathrm{~d}}$ (if we except $n=1$, then these time-intervals vary from 1.5 to 2 years), while the events around 1986 and 1991 occur at longer time-intervals from maxima of $R_{n}^{3 \mathrm{~d}}$ (4-6.5 years). The same results are indicated by the row of averaged scaled time-intervals. We can consider the geomagnetic jerks of 1986 and 1991 more localized events. These results, especially for the events of 1991 and 1999, are different from those of Pinheiro et al. (2011). According to their results the 1991 event is a global one, while the 1999 event is a local one.

\section{Discussion and conclusions}

Understanding the origin of rapid changes of the geomagnetic field arising from inside the Earth, such as the geomagnetic jerks, is challenging. The recent joint analysis of ground-based and satellite data has brought some progress, mainly because of their very different distributions in space and in time. Nevertheless, such new data are available only over the last decade, and there is a clear need to apply new mathematical techniques to geomagnetic series covering longer periods.

Here, we show that a specific behavior of geomagnetic jerks can be noted mostly in different longitude belts. Particular events, having as signatures strong fields of the rms $d_{1}$ coefficients, are not extended over the whole globe. As shown by the available animation in the supplemental material, starting with the 1901 event, the strong field is concentrated mostly in four longitudinal belts. The known extended 1913 jerk is represented by a strong field during 1910-1911, while the one in 1925 is represented by a strong field in four large longitudinal belts (the largest one in the center). An event around 1932 is presented by a strong field in the longitudinal belts from 1930-1932. The event of 1949 is characterized by a strong field that lasts for the longest period of time (1945-1951), covering almost half of the globe. The well known geomagnetic jerk in 1969 is presented by a spot over Europe and an eastern belt of strong fields during 1968-1969, followed by two large belts of strong field during 1970-1971 and a relatively strong field in the Southern hemisphere in 1972. The 1978 geomagnetic jerk is shown 
Table 2. Geomagnetic jerk dates correspondences to the $R_{n}^{3 \mathrm{~d}}$ maxima.

\begin{tabular}{|c|c|c|c|c|c|c|c|c|c|c|c|c|c|c|}
\hline & $n=1$ & $n=2$ & $n=3$ & $n=4$ & $n=5$ & $n=6$ & $n=7$ & $n=8$ & $n=9$ & $n=10$ & $n=11$ & $n=12$ & $\begin{array}{l}\text { Averaged } \\
\text { time-intervals } \\
\text { (year) }\end{array}$ & $\begin{array}{l}\text { Averaged } \\
\text { scaled } \\
\text { time-intervals }\end{array}$ \\
\hline 1969.5 & $\begin{array}{l}0.850 \\
-13\end{array}$ & $\begin{array}{l}0.207 \\
-0.5\end{array}$ & $\begin{array}{l}0.019 \\
-0.5\end{array}$ & $\begin{array}{l}0.332 \\
4.5\end{array}$ & $\begin{array}{l}0.831 \\
-0.5\end{array}$ & $\begin{array}{l}0.697 \\
-0.5\end{array}$ & $\begin{array}{l}0.958 \\
2\end{array}$ & $\begin{array}{l}0.811 \\
2\end{array}$ & $\begin{array}{l}0.944 \\
2\end{array}$ & $\begin{array}{l}0.634 \\
2\end{array}$ & $\begin{array}{l}0.479 \\
2\end{array}$ & $\begin{array}{l}0.150 \\
2\end{array}$ & 2.791 & 7.186 \\
\hline 1978.5 & $\begin{array}{l}0.850 \\
-4\end{array}$ & $\begin{array}{l}0.221 \\
-4\end{array}$ & $\begin{array}{l}0.344 \\
-4\end{array}$ & $\begin{array}{l}0.391 \\
1\end{array}$ & $\begin{array}{l}0.574 \\
-1.5\end{array}$ & $\begin{array}{l}0.713 \\
-1.5\end{array}$ & $\begin{array}{l}0.181 \\
1\end{array}$ & $\begin{array}{l}0.173 \\
1\end{array}$ & $\begin{array}{l}0.726 \\
1\end{array}$ & $\begin{array}{l}0.178 \\
-1.5\end{array}$ & $\begin{array}{l}0.402 \\
1\end{array}$ & $\begin{array}{l}0.650 \\
1\end{array}$ & 1.876 & 5.57 \\
\hline 1986.5 & $\begin{array}{l}0.802 \\
4\end{array}$ & $\begin{array}{l}0.199 \\
4\end{array}$ & $\begin{array}{l}0.815 \\
4\end{array}$ & $\begin{array}{l}0.541 \\
1.5\end{array}$ & $\begin{array}{l}0.286 \\
-3.5\end{array}$ & $\begin{array}{l}0.766 \\
6.5\end{array}$ & $\begin{array}{l}0.725 \\
4\end{array}$ & $\begin{array}{l}0.112 \\
4\end{array}$ & $\begin{array}{l}0.636 \\
4\end{array}$ & $\begin{array}{l}0.692 \\
6.5\end{array}$ & $\begin{array}{l}0.350 \\
4\end{array}$ & $\begin{array}{l}0.272 \\
-8.5\end{array}$ & 4.541 & 12.757 \\
\hline 1991.5 & $\begin{array}{l}0.907 \\
-6\end{array}$ & $\begin{array}{l}0.761 \\
-6\end{array}$ & $\begin{array}{l}0.913 \\
-6\end{array}$ & $\begin{array}{l}0.330 \\
-3.5\end{array}$ & $\begin{array}{l}0.215 \\
1.5\end{array}$ & $\begin{array}{l}0.766 \\
+11.5\end{array}$ & $\begin{array}{l}0.725 \\
9\end{array}$ & $\begin{array}{l}0.835 \\
-8.5\end{array}$ & $\begin{array}{l}0.395 \\
-1\end{array}$ & $\begin{array}{l}0.690 \\
-11\end{array}$ & $\begin{array}{l}0.248 \\
-8.5\end{array}$ & $\begin{array}{l}0.272 \\
-3.5\end{array}$ & 6.333 & 11.823 \\
\hline 1999.5 & $\begin{array}{l}0.907 \\
+2\end{array}$ & $\begin{array}{l}0.526 \\
2\end{array}$ & $\begin{array}{l}0.913 \\
2\end{array}$ & $\begin{array}{l}0.023 \\
-0.5\end{array}$ & $\begin{array}{l}0.761 \\
-0.5\end{array}$ & $\begin{array}{l}0.895 \\
-3\end{array}$ & $\begin{array}{l}0.865 \\
-3\end{array}$ & $\begin{array}{l}0.835 \\
-0.5\end{array}$ & $\begin{array}{l}0.851 \\
-0.5\end{array}$ & $\begin{array}{l}0.690 \\
-3\end{array}$ & $\begin{array}{l}0.361 \\
-0.5\end{array}$ & $\begin{array}{l}0.166 \\
-0.5\end{array}$ & 1.5 & 3.945 \\
\hline Sum & $\begin{array}{l}33.81 \\
29\end{array}$ & $\begin{array}{l}52.30 \\
16.5\end{array}$ & $\begin{array}{l}51.61 \\
16.5\end{array}$ & $\begin{array}{l}51.23 \\
11\end{array}$ & $\begin{array}{l}23.09 \\
7.5\end{array}$ & $\begin{array}{l}29.67 \\
23\end{array}$ & $\begin{array}{l}29.01 \\
19\end{array}$ & $\begin{array}{l}54.74 \\
16\end{array}$ & $\begin{array}{l}12.90 \\
8.5\end{array}$ & $\begin{array}{l}41.26 \\
24\end{array}$ & $\begin{array}{l}53.75 \\
16\end{array}$ & $\begin{array}{l}62.00 \\
15.5\end{array}$ & $\begin{array}{l}\leftarrow \text { Sum of Sca } \\
\leftarrow \text { Sum of Nol }\end{array}$ & $\begin{array}{l}\text { ime-intervals } \\
\text { led time-intervals }\end{array}$ \\
\hline
\end{tabular}

by local foci of strong field over some regions of the Earth. Finally, the event in 1986 is represented by a strong field mostly over the Southern African and the South Pole region. Apart from these events corresponding to geomagnetic jerks already noted in literature, the $d_{1}$ coefficients indicate additional particular events, especially in 1917-1918, 19451946, 1952-1954, 1963-1965, so far not reported as possible geomagnetic jerks.

Recently, Olsen and Mandea (2008) have shown that changes in the core magnetic field can be as short as a few months. These rapid secular variation fluctuations are not globally observed from satellite data. Our results based on both observatory and synthetic data are a complement of previous studies investigating the geomagnetic jerks or the spatial distribution of rapid secular variation fluctuations, and illustrate, with results covering nearly one century, that these events are not global in appearance. Also the unbalanced contributions of the spherical harmonic degrees at the different jerks are intriguing and deserve deeper attention in further studies and analyses. To conclude, all these findings are important for continuing the present investigations on jerks to uncover more details and features of the core dynamics.

\section{Appendix A}

\section{The STFT definition}

The Short Time Fourier Transform (STFT) of onedimensional continuous time function $x(t)$ is the Fourier Transform of the function $x(t)$ multiplied by a window function, where the window is slid along the time axis, resulting in a two-dimensional representation of the signal ( Jacobsen and Lyons, 2003):

$X(\tau, \omega)=\int_{-\infty}^{\infty} x(t) w(t-\tau) e^{-j \omega t} d t$

where $w(t)$ is the window function, commonly a Gaussianform centered around zero. Generally, $X(\tau, \omega)$ is a complex function representing the phase and magnitude of the signal over time and frequency. The magnitude squared of the STFT yields the spectrogram of the function:

$\operatorname{spectrogram}\{x(t)\} \equiv|X(\tau, \omega)|^{2}$.

In the discrete time case, the data to be transformed are broken up into blocks, which usually overlap each other. Each block is Fourier transformed, and the complex result is added to a matrix, which records magnitude and phase for each point in time and frequency. This can be expressed as:

$\operatorname{STFT}\{x[m]\} \equiv X(k, \omega)=\sum_{m=-\infty}^{\infty} x[m] w[m-k] e^{-j m t_{0} \omega}$,

likewise, with discrete signal $x[m]$ and discrete window $w[m]$, while the frequency $\omega=2 \pi f$ is continuous. But in most typical applications the STFT is performed on a computer using the Fast Fourier Transform algorithm, so both variables are discrete and quantized: $m=0,1,2, \ldots N-1$ and $f=n \cdot f_{0}=n \cdot f_{s} / N=n /\left(t_{0} \cdot N\right)(n=0,1,2, \ldots, N-1)$. Then the STFT is defined as:

$\operatorname{STFT}\{x[m]\} \equiv X(k, n)=\sum_{m=0}^{N-1} x[m] w[m-k] e^{-j 2 \pi m n / N}$, 
where $k=0,1,2, \ldots, N-1$, and the spectrogram is defined as:

$\operatorname{spectrogram}\{x[m]\} \equiv|X(k, n)|^{2}$.

The MatLlab function:

$\mathbf{B}=\operatorname{specgram}\left(\boldsymbol{x}, \quad n f f t, f_{s}\right.$, window(length), numoverlap)

calculates the windowed discrete-time Fourier transform for the signal in vector $\boldsymbol{x}$ with length $N$; nfft specifies the FFT length that specgram uses, $f_{s}$ specifies the sampling frequency, window specifies a windowing function and the number of samples specgram uses in its sectioning of vector $\boldsymbol{x}$. If $\boldsymbol{x}$ is real, specgram computes the discrete-time Fourier transform at positive frequencies only. If $N$ is even, specgram returns $n f f t / 2+1$ rows in $\mathbf{B}$ (including the zero and Nyquist frequency terms). If $n$ is odd, specgram returns $(n f f t+1) / 2$ rows. The number of columns in $\mathbf{B}$ is the integer number (fix number) of ( $N$-numoverlap)/(length(window)numoverlap)).

The windows of the spectrogram figure present in timefrequencies axis the plots of the scaled logarithmic (in $\mathrm{dB}$ ) amplitudes: $20 * \log _{10}(\operatorname{abs}(\mathbf{B}))$.

\section{Appendix B}

\section{WARMA - noise generation}

"warma" is a colored AR(3) noise generated by MATLAB from the formula:

$b_{2}(t)=-1.5 b_{2}(t-1)-0.75 b_{2}(t-2)-0.125 b_{2}(t-3)+b_{1}(t)+0.5$, $b_{1}(t)$ being a uniform white noise series.

The test of normal probability plot shows that the underlying distribution of the "warma" series for $t=1,2,3 \ldots, 1000$, is a normal one, with variance $=0.6135$ and sigma $=0.78326$.

The following changes on "warma" signal provide a more realistic noise (more like the noise of a secular variation signal). From a 125-value long colored noise [warma(1:125), variance $=0.6079$, sigma $=0.7797)]$ we generate a more extended noise (1000 value long), where 8 values between each couple of successive values are generated by adding proportionally the difference between these successive values. The test of normal probability plot shows a slight deviation from the normal distribution, with variance $=0.2284$ and sigma $=0.4779$. Each value is multiplied by certain number such that the amplitude of the noise reaches about $15 \%$ of the signal amplitude (the signal composed by differences of several exponential spikes like (1)).
Acknowledgements. We would like to thank F. J. Lowes, V. Lesur (topic editor of SED), R. Holmes, S. Macmillan and A. Chambodut, for their constructive and helpful comments regarding the previous versions of this paper.

Edited by: C. A. Jones

\section{Supplementary material related to this article is available online at: http://www.solid-earth.net/3/131/ 2012/se-3-131-2012-supplement.zip.}

\section{References}

Alexandrescu, M., Gilbert, D., Hulot, G., Le Mouël, J. -L., and Saracco, G.: Detection of geomagnetic jerks using wavelet analysis, J. Geophys. Res., 100, 12557-12572, 1995.

Alexandrescu, M., Gilbert, D., Hulot, G., Le Mouël, J. -L., and Saracco, G.: Worldwide wavelet analysis of geomagnetic jerks, J. Geophys. Res., 101, 21975-21994, 1996.

Backus, G., Parker, R., and Constable, C.: Foundation of Geomagnetism, Cambridge University Press, 103 pp., 1996.

Bloxham, G., Zatman, S., and Dumberry, M.: The origin of geomagnetic jerks, Nature, 420, 65-68, 2002.

Brockwell, P. J. and Davis, R. A.: Time series: Theory and Methods, 2nd Edn., Springer, 2009.

Chambodut, A. and Mandea, M.: Evidence for geomagnetic jerks in comprehensive models, Earth, Planets and Space, 57, 139-149, 2005.

Chambodut, A., Panet, I., Mandea, M., Diament, M., Holschneider, M., and James, O.: Wavelet frames: an alternative to spherical harmonic representation of potential fields, Geophys. J. Int., 163, 3, 875-899, 2005.

Chau, H. D., Ducruix, J., and Le Mouël, J. -L.: Sur le caractère planétaire du saut de variation séculaire de 1969-1970, C. R. Acad. Sci. Paris, B293, 157-160, 1981.

Courtillot, V., Ducruix, J., and Le Mouël, J. -L.: Sur une accélérations récente de la variation séculaire du champ magnétique terrestre, C. R. Acad. Sci. Paris, D287, 1095-1098, 1978.

Daubechies, I.: Ten lectures on wavelets, CBMS-NSF Regional Conference Series in Applied Mathematics, Society for Industrial and Applied Mathematics, Philadelphia, 1992.

Gabor, D.: Theory of communications, J. Inst. Elec. Eng., 93, 429457, 1946.

Grossmann, A., Holschneider, M., Kronland-Martinet, R., and Morlet, J.: Detection of abrupt changes in sound signals with the help of wavelet transform, in: Inverse Problems: An Interdisciplinary Study, Adv. Electron. Electron. Phys., 19, San Diego, CA: Academic, 298-306, 1987.

Holme, R., Olsen N., and Bairstow F. L.: Mapping geomagnetic secular variation at the core-mantle boundary, Geophys. J. Int., 186, 521-528, 2011.

Holschneider, M.: Wavelets: An Analysis Tool, Oxford: Oxford University Press, 1995. 
Jacobsen, E. and Lyons, R.: The sliding DFT, Signal Processing Magazine, 20, 2, 74-80, 2003.

Jackson, A., Jonkers, A. R. T., and Walker, M. R.: Four centuries of geomagnetic secular variation from historical records, Phil. Trans. R. Soc. Lond., 358, 957-990, 2000.

Kumar, P. and Georgiu, E. F.: Wavelet Analysis in Geophysics: An Introduction, in Wavelet Analysis and its Applications, 1-43, Academic Press, 1994.

Le Huy, M., Alexandrescu, M., Hulot, G., and Le Mouël, J.-L.: On the characteristics of successive geomagnetic jerks, Earth Planets Space, 50, 723-732, 1998.

Lowes, F. J.: Spatial power spectrum of the main geomagnetic field, and extrapolation to the core, Geophys. J. R. astr. Soc., 36, 717$730,1974$.

Lowes, F. J.: Spatial Geomagnetic Spectrum, in Encyclopedia of geomagnetism and paleomagnetism, edited by: Gubbins, D. and Herrero-Bervera, E., Springer, 351-353, 2007.

MATLAB: the language of technical computing, Using MATLAB, MathWorks Inc., 2004.

Mandea, M., Bellanger, E., and Le Mouël, J. -L.: A geomagnetic jerk for the end of the 20th century?, Earth Planet. Sci. Lett., 183, 369-373, 2000.

Mandea, M., Holme, R., Pais, A., Pinheiro, K., Jackson, A., and Verbanac, G.: Geomagnetic Jerks: Rapid Core Field Variations and Core Dynamics, Space Sci. Rev., 155, 147-175, 2010.

Meyer, Y.: Wavelets and Operators, Cambridge University Press, 1992.

Meyer, Y.: Wavelets, Algorithms and Applications, SIAM, Philadelphia, 1993.
Misiti, M., Misiti, Y., Oppenheim, G., and Poggi, J. M.: Wavelets and Their Applications, Hermes Lavoisier, ISTE Publishing Knowledge, 2007.

Nagao, H., Iyemori, T., Higuchi, T., and Araki, T.: Lower mantle conductivity anomalies estimated from geomagnetic jerks, J. Geophys. Res., 108, 2254, doi:10.129/2002JB001786, 2003.

Olsen, N. and Mandea, M.: Investigation of a secular variation impulse using satellite data: The 2003 geomagnetic jerk, Earth Planet. Sc. Lett., 255, 94-105, 2007.

Olsen, N. and Mandea, M.: Rapidly changing flows in the Earth's core, Nat. Geosci., 1, 390-394, 2008.

Pinheiro, K. J., Jackson A., and Finlay C. C.: Measurements and uncertainties of the occurrence time of the 1969, 1978, 1991 and 1999 geomagnetic jerks, Geochem. Geophys. Geosyst., 12, Q10015, doi:10.1029/2011GC003706, 2011.

Sabaka, T. J., Olsen, N., and Langel, R. A.: A comprehensive model of the quiet-time, near-Earth magnetic field: Phase 3, Geophys. J. Int., 151, 32-68, 2002.

Sabaka, T. J., Olsen, N., and Purucker, M. E.: Extending comprehensive models of the Earth's magnetic field with Ørsted and CHAMP data, Geophys. J. Int., 159, 521-547, 2004.

Stewart, D. N. and Whaler, K. A.: Geomagnetic disturbance fields: an analysis of observatory monthly means, Geophys. J. Int., 108, 215-223, 1992.

Wardinski I. and Holme R.: De-noising data for secular variation studies, Geophys. I. Int., 185, 2, 653-662, 2011. 